\title{
QTL Validation and Development of SNP-based High Throughput Molecular Markers Targeting a Genomic Region Conferring Narrow Root Cone Angle in Aerobic Rice Production Systems
}

\section{Ricky Vinarao}

The University of Queensland School of Agriculture and Food Sciences

\section{Christopher Proud}

The University of Queensland School of Agriculture and Food Sciences

\section{Peter Snell}

NSW DPI: New South Wales Department of Primary Industries

\section{Shu Fukai}

The University of Queensland School of Agriculture and Food Sciences Jaquie Mitchell ( $\nabla$ jaquie.mitchell@uq.edu.au )

The University of Queensland School of Agriculture and Food Sciences https://orcid.org/0000-00017641-7935

\section{Research Article}

Keywords: QTL validation, KASP, molecular markers, aerobic production, rice, root angle

Posted Date: June 28th, 2021

DOI: https://doi.org/10.21203/rs.3.rs-636404/v1

License: (c) (i) This work is licensed under a Creative Commons Attribution 4.0 International License.

Read Full License 


\section{Abstract}

\section{Background}

Aerobic rice production (AP) is a potential solution to the imminent global water crisis as it consumes less water than traditional permanent water culture. Narrow root cone angle (RCA) and subsequent development of deeper rooting systems are considered AP adaptation traits by enabling water uptake at depth. Quantitative trait loci (QTL), such as $q R C A 4$, contributing to RCA variation in AP systems have been previously identified. However, their usefulness depends on validation in a range of genetic backgrounds, development of associated molecular markers, and identification of possible donors in other subpopulations.

Results

Using three $\mathrm{F}_{2}$ bi-parental populations derived from IRAT109 crossed with three different genetic backgrounds, and genotypes with known $q R C A$ composition, QTL validation were performed and molecular markers developed. qRCA4 was shown to be effective in multiple backgrounds, explaining 9.3$17.3 \%$ of the genotypic variation in the populations tested and the introgression of the favourable allele resulted in 11.7-15.1 ${ }^{\circ}$ narrower RCA. Novel kompetive allele specific PCR (KASP) molecular markers have been developed associated with narrow RCA. Core molecular marker quality metrics have shown robustness and breeding program specific utility of up to $83 \%$. qRCA4 was further fine mapped into a 720 $\mathrm{kb}$ region. Analysis of candidate genes identified those related with plant response to abiotic stress stimulus together with root development. Available public database search found 178 potential donors representing major rice subpopulations and may be used readily by breeding programs.

\section{Conclusion}

This study validated $q R C A 4$ 's effect in multiple genetic backgrounds further strengthening its value in improvement of rice genotypes for AP adaptation. Furthermore, the development of novel KASP markers has ensured the opportunity for its seamless introgression across pertinent breeding programs. Future research in metabolomic and transcriptomic analysis of QTL positive genotypes should improve the understanding of the physiological mechanisms underpinning narrow RCA and development of deeper rooting. Finally, this work provides the tools and opportunity to accelerate the development of genotypes with narrow RCA through marker assisted selection in breeding programs targeting AP, which may ultimately contribute to more sustainable rice production where water availability is limited.

\section{Background}

To feed the increasing number of rice consumers by 2030 , world rice production has to increase by $40 \%$ (Khush 2005). This is a tall order considering the devastating negative effects of climate change such as looming water crisis brought about by climate change, increasing population, and higher food demand (Cllinttat al 201 1) Anmbin rinn nmaduntinn /ADI nuntam is a promising technology which may provide Loading [MathJax]/jax/output/CommonHTML/fonts/TeX/fontdata.js 
solutions to this conundrum. AP system is an intensive rice farming method consisting of direct seeded rice cultivation under non-flooded conditions and is usually well-irrigated (Kato et al. 2009). Compared with upland cultivation, AP has higher input levels and does not encounter drought (Kato and Katsura 2014). Although water availability in AP system is generally high, transient water deficit may develop between irrigation events. This is distinct from water deficit as commonly perceived in relatively low yielding upland cultivation.

Several physiological and morphological attributes have been postulated to minimise effect of intermittent water deficit and enable stable or increased rice yield in AP systems. These include (1) vigorous biomass production with harvest index ranging from 0.40-0.48, (2) improved yield component traits such as increased number of spikelets per unit area, (3) plants with intermediate height $(<130 \mathrm{~cm})$, larger and thicker tillers and leaves, and (4) improved root system architecture (RSA) such as increased rooting depth (Kato and Katsura 2014). RSA is the arrangement of the crop root system in terms of specific geometric configuration across a rooting medium, and is particularly important as it determines anchorage, soil nutrient and water exploitation, and developmental plasticity, which will ultimately affect maximum yield and yield stability. One of the components of RSA is rooting angle - root cone angle (RCA), is the plant's root angle relative to the vertical axis. Genotypes with narrow RCA tend to have deeper rooting systems (Uga et al. 2013a) and is therefore highly relevant for AP adaptation. Several experiments in upland field conditions with drought stress have shown the advantage given by narrow RCA. Using 12 cultivars, it was shown that narrow RCA was associated with development of deeper roots (Kato et al. 2006)). Similarly, genotypes with higher ratio of deep roots (RDR, narrow RCA) tended to have increased rooting depth in an upland field condition with drought stress (Uga et al. 2013a). The advantage of having deeper roots in an AP system was indicated with positive genetic correlations observed between percentage of deep roots and grain yield when a set of 20 diverse cultivars were evaluated (Mitchell et al. 2019). To facilitate the development of genotypes with narrower RCA and deeper rooting and their further inclusion in breeding programs, identification of genes and quantitative trait loci (QTL) governing these traits is imperative.

To date, a number of studies conducted in paddy fields or in transplanted systems identified genomic regions associated with rooting angle. Using Kinandang Patong, several deep rooting QTL (DRO1, DRO2, DRO3, DRO4, and DRO5) located in different chromosomes were identified (Kitomi et al. 2015; Uga et al. 2015; Uga et al. 2013a; Uga et al. 2013b). Of these, only DRO1 was cloned and was shown to increase rooting depth of genotypes with favourable allele, thereby stabilising rice yield in upland conditions and under drought stress. IRAT109, an upland tropical japonica (tropjap) genotype, was also utilised to map QTL for RDR in transplanted systems, identifying $q R D R-2$ as a genomic region located in chromosome 2 (Lou et al. 2015). Recently, novel, stable, consistently identified across environments and environment-specific genomic regions conferring narrow RCA were identified in AP systems (Vinarao et al. 2021). qRCA4 was found to be a major and stable QTL identified across all environments tested, and $q R C A 1.1$ was a novel QTL which was also expressed in all environments. Of the environment specific QTL, $q R C A 2.1$ and $q R C A 2.2$ were found to be associated in intermittent water stress Loading [MathJax]/jax/output/CommonHTML/fonts/TeX/fontdata.js h effects. In order to facilitate the introgression 
of these QTL in relevant breeding programs, it is imperative to demonstrate the effect of these genomic regions in multiple genetic backgrounds (Cobb et al. 2019). Additionally, molecular markers tagging these QTL, and ultimately the target trait - narrow RCA and deeper roots will also be vital for seamless introgression of these loci into breeding programs.

A number of molecular markers have been developed and used extensively in rice improvement efforts (Collard and Mackill 2008; Mackill and McNally 2004) including restriction fragment length polymorphism, random amplified polymorphic DNA, cleaved-amplified polymorphic sequence, simple sequence repeat (SSR), and single nucleotide polymorphism (SNP) markers. Numerous SSR markers were developed by McCouch et al. (2002) and their corresponding sequences are available online (https://archive.gramene.org/markers/). SSRs are most widely used markers in major cereals crops as they are highly reproducible, co-dominant, relatively simple and cheap to use, and generally highly polymorphic. SSRs do come with some disadvantages such that they generally give information to a single locus only per assay, have difficulty in merging data across platforms and cumbersome curation of allele sizes into databases. With the advent of whole genome sequence information, SNPs became the marker of choice for majority of high throughput genotyping applications because of (1) they are ubiquitous in eukaryotic genomes, (2) cost effectiveness in terms of assay and automation, and (3) simple allele calling, data analysis and curation into databases due to their bi-allelic nature (Thomson et al. 2017). Recently, high-density SNP chip called the Cornell-IR RiceLD Array have been developed at the International Rice Research Institute (IRRI) in collaboration with Cornell University (https://isl.irri.org/services/genotyping/7k). This chip allows for the simultaneous genotyping of a sample across 7,000 SNP loci including 21 gene-based SNP diagnostic for submergence tolerance SUB1A gene, grain quality characteristics, and loci for resistance to bacterial leaf blight, blast, brown planthopper, and tungro. One disadvantage of this SNP chip is that it is fixed (SNP markers and traits), similar to an amplicon-based SNP genotyping assay developed recently (Arbelaez et al. 2019a). On the other hand, there are a number of available high-throughput technologies able to run flexible sets of SNP markers. Kompetitive allele-specific polymerase chain reaction (KASP) marker assay is a single-step genotyping technology that uses two competing allele-specific forward primers, one reverse primer, and a master mix with a fluorescence resonance energy transfer cassette and Taq DNA polymerase. With this, pre-identified, co-dominant alleles for SNPs and insertion/deletion variants between parents, progeny, and other genotypes being tested are determined (Semagn et al. 2014). The Genotyping Services lab of IRRI have developed and validated trait based KASP SNP markers for the following: yield components (grain number, grain size, and panicle architecture), grain quality (amylose, chalk, and gelatinization temperature), abiotic stress tolerance (anaerobic germination, drought, cold, and submergence), and disease and pest resistance (bacterial blight, blast, brown planthopper, and tungro) (https://isl.irri.org/services/genotyping/trait-based-genotyping). To our knowledge, KASP SNP markers targeting root angle, whether in conventional flooded or AP systems, have not been developed and validated to date. Using genotypes with known $q R C A$ composition and newly developed segregating populations, this study was carried out to validate the effect of $q R C A$ QTL across several genetic harkarnunds and to develon $K \triangle S P$ hased SNP markerc targeting qRCA QTL for its precise introgression Loading [MathJax]/jax/output/CommonHTML/fonts/TeX/fontdata.js 
into various genetic backgrounds pertinent to rice breeding programs, particularly targeting environments exposed to transient water deficit. Additionally, this study also determined molecular marker quality metrics of the newly developed markers and identified potential donors using publicly available databases.

\section{Methods}

\section{Plant materials}

To validate the effect of target QTL in different genetic backgrounds, progenies were produced between IRAT109 and three recipient parents (RP), RL11, Langi, and Norin PL8. $F_{1} s$ were produced by crossing IRAT109 with RPs, and subsequently, $F_{2}$ progenies were produced by selfing $F_{1}$ s. A total of $810 F_{2}$ progenies were genotyped and evaluated for RCA. Of these, 283, 196, and 331 were derived from crosses between IRAT109/Norin PL8 (IRNO), IRAT109/Langi (IRLA), and IRAT109/RL11 (IRRL), respectively. A total of 60 genotypes were utilised to test the molecular marker quality metrics of the newly developed markers. Of these, six were either released varieties or donors of high importance to the Australian breeding program - IRAT109, Sherpa, Reiziq, Langi, RL11, and Norin PL8. The other 54 genotypes were recombinant inbred lines (RILs) derived from Sherpa/IRAT109 and were previously identified to have varying combinations of QTL for RCA (Vinarao et al. 2021).

\section{Phenotyping for RCA}

Three experiments were carried out to evaluate the segregating populations developed above, along with the six varieties/checks following the clear pot method described by Vinarao et al. (2021), with some modifications, at the University of Queensland (UQ) St Lucia $\left(27.4975^{\circ} \mathrm{S}, 153.0137^{\circ} \mathrm{E}\right)$ campus. Briefly, single seeds of $F_{2} s$ and check genotypes were direct seeded in $4 \mathrm{~L}$ clear pots (ANOVApot ${ }^{\circledR}, 200 \mathrm{~mm}$ diameter, $190 \mathrm{~mm}$ height, http://www.anovapot.com/php/anovapot.php) filled with pine bark potting media (70\% composted pine bark $0-5 \mathrm{~mm}, 30 \%$ coco peat, $\mathrm{pH} 6.35, \mathrm{EC}=650 \mathrm{ppm}$, nitrate $=0$, ammonia < $6 \mathrm{ppm}$ and phosphorus $=50 \mathrm{ppm})$ with $3 \mathrm{~g} / \mathrm{L}$ Osmocote Exact 3-4M (19-9-10 + 2MgO + TE, ICL Specialty Fertilizers), 2g/L Osmocote Exact 5-6M (15-9-12 + 2MgO + TE) and 0.82g/L Suscon Maxi Green (Nufarm, Australia). Using a pair of forceps, seeds were sown vertically at a depth of $3 \mathrm{~cm}$ along the pot wall with a final density of 12 plants per pot. In every experiment, parental genotypes for each specific $F_{2}$ population were replicated in every pot, while the other check genotypes were replicated every incomplete block (four clear pots/incomplete block). To prevent penetration of light, similar sized black pots were used to cover the clear pots. Pots were placed on a table and a constant layer of water $(\sim 4 \mathrm{~cm}$ from the base of the inner pot) was maintained to mimic aerobic growing conditions. The experiments were carried out in a temperature-controlled glasshouse set at $28^{\circ} \mathrm{C} / 21^{\circ} \mathrm{C}$ day/night temperatures with natural light. RCA were measured manually 35,42 , and 49 days after sowing (DAS) using a protractor, by measuring the cone angle between the two most external nodal roots.

\section{Development of KASP SNP molecular markers}


SNP variations across target QTL regions were investigated using the 3K Rice Genome Project (3KRGP) database which included IRAT109, the main donor genotype in this study as well as six varieties relevant to the Australian breeding program (M7, M102, M401, M203, Calrose 76, and YRM6-2). For qRCA4, SNPs unique to IRAT109 were identified along the region, $\pm 100 \mathrm{~kb}$ upstream and downstream the QTL's confidence interval. Using the SNPs identified, linkage disequilibrium (LD) in the region was investigated using TASSEL (Bradbury et al. 2007) by computing the standardised disequilibrium coefficient (D') from the full matrix, in both the japonica sub-group and all the genotypes included in 3KRGP. Additionally, allele frequencies of the selected SNPs were also determined using the SNP-Seek database (Mansueto et al. 2017). Their relative allele frequencies were noted in different populations: tropjap, subtropical japonica (subtrop), temperate japonica (tempjap), and in all 3KRGP (japonica, indica, and admixed, all3K). SNPs having the desired allele frequencies were selected and analysed further. DNA sequence surrounding the SNP of interest ( $\pm 200 \mathrm{bp}$ ) were downloaded from the SNP-Seek database and multiple sequence alignment was carried out using CLUSTALW implemented in Molecular Evolutionary Genetic Analysis X (Kumar et al. 2018) software to ascertain that the region surrounding the target SNP was fairly conserved or with minimal variation. Finally, the downloaded IRAT109 sequence was aligned through basic local alignment search tool with the Nipponbare sequence implemented in the Rice Annotation Project Database (RAP-DB) to confirm that the regions selected were unique and only occurred in the target chromosome. SNPs and their surrounding sequences which passed the criteria described above were compiled and sent to Intertek, Australia (https://www.intertek.com/agriculture/agritech/) for subsequent development of KASP molecular markers. To constitute a plate to validate newly designed molecular markers, IRAT109 and Sherpa were replicated four times while Reiziq, Langi, RL11, and Norin PL8 were replicated thrice. Leaf samples of IRAT109 were also combined with other genotypes (Sherpa, Reiziq, Langi, RL11, and Norin PL8) to create heterozygotes (a total of 20 samples). To complete the set, leaf samples of the 54 RILs derived from IRAT109/Sherpa were also included, bringing the total to 94. Two 4$6 \mathrm{~mm}$ leaf samples were sampled and placed per well of an Abgene ${ }^{\mathrm{TM}} 96$ deepwell storage plate (ThermoFisher Scientific, USA) to constitute the validation plate. Leaf samples were subsequently freezedried at $-55^{\circ} \mathrm{C}$ for $48 \mathrm{~h}$ using Alpha 1-2 LDplus (Martin Christ, Germany) and then sealed using 96-well sealing mats (ThermoFisher Scientific, USA). Upon selection of well-performing markers, a similar method was used to prepare the $F_{2}$ segregating genotypes. KASP genotyping of validation genotypes and segregating population was carried out at the Intertek, Australia laboratory following standard manufacturer specifications.

\section{Molecular marker and QTL validation}

Cluster graph for each SNP genotyping assay was visualised using KlusterCaller ${ }^{\text {TM }}$ and SNPviewer software (LGC Biosearch Technologies, UK). In some instances where automated SNP genotype calling of the software classified some individuals into 'uncallable' but were clearly belonging into a cluster, these were manually called into their respective cluster genotypes. Newly developed KASP molecular markers were evaluated for core quality metrics as described by Platten et al. (2019), which included call rate, false positive rate (FPR), false negative rate (FNR) and utility. FPR was the proportion (percent, \%) of 
known genotypes (recipients) which were incorrectly classified as having the QTL, and was computed with the following equation:

$$
F P R(\%)=\frac{\# \text { ofrecipientswithfavourableallele }}{\text { Total\# ofknownrecipients }} \times 100
$$

On the other hand, FNR was the proportion of donor genotypes (known to harbour QTL) which were incorrectly classified to not possess the QTL due to not having the favourable allele of a marker, and was given by the following equation:

$$
F N R(\%)=\frac{\# \text { ofdonorswithunfavourableallele }}{\text { Total\# ofknowndonors }} \times 100
$$

To estimate these molecular marker metrics, a set of 60 genotypes identical to the genotypes specified above were assembled and genotyped using the newly designed KASP markers targeting qRCA4, qRCA1.1, qRCA2.1, and qRCA2.2. Of the 54 RILs, 23, 22, 21, and 30 of them had qRCA4, qRCA1.1, qRCA2.1, and $q R C A 2.2$, respectively. Based on the QTL composition of the RILs, FPR and FNR of the individual markers were analysed and were subsequently used to select markers used to genotype the segregating $F_{2}$ populations for succeeding QTL validation.

Three $F_{2}$ populations: IRLA, IRNO and IRRL were genotyped with selected markers to validate the effect of qRCA QTL across genetic backgrounds. Composite interval mapping was carried out to validate the effect of $q R C A 4$ across multiple genetic backgrounds, and then stepwiseqtl, refineqtl, and fitqtl functions in R/qtl were used to estimate the QTL effect (Broman et al. 2003). Bayesian credible confidence interval was determined using bayesint function of R/qtl. Single marker analysis (SMA) was carried out in R ( Core Team 2019) to test for the association of marker with RCA in case of qRCA1.1, qRCA2.1, and qRCA2.2 and $\mathrm{R}^{2}$ was computed to estimate the proportion of phenotypic variation contributed by individual markers to the phenotype.

\section{Identification of candidate genes and possible donors present in 3KRGP}

Using the new confidence interval calculated for $q R C A 4$, details of the annotated genes located within the region were batch downloaded from RAP-DB which included information such as gene ID, gene function, strand, physical position, and gene ontology (Sakai et al. 2013). Possible candidate genes controlling qRCA4-mediated reduction in RCA were identified based on the gene function along with ontology, giving priority with those involved in root development as well as those related with response to abiotic stress stimulus.

Allele frequencies of the candidate SNP molecular markers were investigated using the 3KRGP data available online (Mansueto et al. 2017). Genotypes possessing the favourable qRCA4 allele were identified in each subpopulation which may represent donors for breeding programs dealing with specific 


\section{Statistical analysis}

A multiplicative mixed linear model was carried out for the analysis of the phenotype data per population which was executed in ASReml (V4.1; VSNi, UK) package running in the R environment (R Core Team 2019). Parents of the respective $F_{2}$ population and other checks were treated as fixed effects while the $F_{2}$ plants were treated as random effects. Best linear unbiased predictors were then calculated after accounting for the randomisation and other blocking parameters. Heritability for each experiment $\left(\mathrm{F}_{2}\right.$ population) was computed as specified by Smith et al. (2006). Additionally, chi-square test for deviation from expected Mendelian $F_{2}$ segregation ratios (1:2:1) of the SNP genotype data was carried out across the three populations to show either conformance or deviation with expected ratios.

\section{Results}

\section{Phenotypic variation of the segregating populations}

Highly significant variation $(P<0.001)$ for RCA was observed in all three $\mathrm{F}_{2}$ populations across each of the three measurement times, with 42 DAS showing the highest heritability ranging from $0.67-0.69$ (Table 1). Highly significant positive correlations were also observed between measurement times in the three populations for IRNO $\left(r=0.61-0.82^{\star \star}\right)$, IRLA $\left(r=0.68-0.87^{\star \star}\right)$, and IRRL $\left(r=0.76-0.89^{\star *}\right)$ populations, respectively with the highest correlation between RCA measured at 42 and 49 DAS for each population. With these results across the populations tested and the relatively high heritability, the data collected from 42 DAS was used in subsequent analyses. 
Table 1

RCA $\left({ }^{\circ}\right)$ statistics of $F_{2}$ s derived from crossing IRAT109 with three recipient parents.

\begin{tabular}{|llll|}
\hline & IRLA & IRRL & IRNO \\
\hline Mean & $n=196$ & $n=331$ & $n=283$ \\
\hline Min & 90 & 98 & 85 \\
\hline Max & 67 & 68 & 63 \\
\hline P value & 124 & 130 & 116 \\
\hline Heritability & $<0.0001$ & $<0.0001$ & $<0.0001$ \\
\hline & 0.69 & 0.67 & 0.68 \\
\hline IRAT109 & Check Genotypes & & 77 \\
\hline Langi & 71 & 79 & 102 \\
\hline RL11 & 111 & 106 & 95 \\
\hline Norin PL8 & ND & 122 & 94 \\
\hline Sherpa & 93 & 98 & 101 \\
\hline Reiziq & 108 & 117 & 117 \\
\hline RCA- root cone angle; IRLA- IRAT109/Langi $F_{2} ;$ IRRL- IRAT109/RL11 $\mathrm{F}_{2} ;$ IRNO- IRAT109/Norin PL8 $\mathrm{F}_{2} ;$ \\
\hline ND- No Data
\end{tabular}

Examining the phenotypic data of the populations, IRRL showed the largest range in terms of RCA, followed by IRLA, and IRNO with the smallest range (Table 1). In terms of the population mean, a similar trend was also observed with IRRL which had a mean RCA of $98^{\circ}$, followed by IRLA with $90^{\circ}$, and then IRNO with $85^{\circ}$. Significant genotypic variation was also observed in the checks included in the evaluation. Of the checks, IRAT109 produced the narrowest RCA ranging from $71-79^{\circ}$. Norin PL8 tended to have relatively narrow RCA ranging from $93-98^{\circ}$. All the other checks (Langi, Reiziq, RL11, and Sherpa) included in the experiments showed relatively wide RCA, ranging from $102-111^{\circ}, 117-124^{\circ}, 95-122^{\circ}$, and $101-117^{\circ}$, respectively.

\section{SNP selection using 3KRGP}

A total of 40 SNPs unique to IRAT109 were identified across the $q R C A 4$ region $\pm 100 \mathrm{~kb}$ of the confidence interval. Initially, all 40 SNPs showed that the average IRAT109 SNP allele frequency was $44.7 \%, 33.9 \%$, $3.6 \%$, and $13.6 \%$ in tropjap, subtrop, tempjap, and all3K, respectively. An LD map was constructed using these 40 SNPs and based on the D' values, the qRCA4 region was found to have three linkage blocks whether the analysis was carried out using the japonica subgroup or all3K genotypes (Fig. S1). This was 
region. After filtering for low allele frequencies, a total of 12 SNPs were retained with an average allele frequency of $24.6 \%, 5.4 \%, 1.5 \%$, and $3.7 \%$ in tropjap, subtrop, tempjap, and all3K, respectively. Marker development for $q R C A 4$ was prioritised since it was shown to be a major genomic region associated with narrow RCA, although SNP markers for other genomic regions were also developed. For qRCA1.1, qRCA2.1, and $q R C A 2.2$, two, one, and one SNP were selected near the peak marker previously reported, respectively. The strategy for selection of SNP for $q R C A 2.2$ was similar to that of $q R C A 4$, while it was a bit different for $q R C A 1.1$ and $q R C A 2.1$, where SNPs selected had moderate allele frequencies in tempjap since the favourable allele for these loci came from Sherpa, a tempjap genotype. In total, a set of 16 SNPs were selected and subsequent KASP marker assays were developed targeting qRCA1.1, qRCA2.1, qRCA2.2, and $q R C A 4$.

\section{Molecular marker development and quality metrics of KASP markers targeting qRCA QTL}

SNP genotype information from the validation plate was processed and visualised to identify wellperforming markers and cull-out poor performing ones. Upon visual inspection, of the 16 markers, two markers - snpOS00936 and snpOS00943 were immediately discarded as they were monomorphic and unable to call heterozygotes, respectively (Fig. S2). On the other hand, molecular markers snp0S00939 and snpOS00948 were polymorphic but showed close clustering among alleles and further inspection was carried out. All other markers showed acceptable clustering and were considered for further analysis. Using the known QTL composition of 54 RILs included in the validation plate, FPR and FNR were computed per molecular marker. From this analysis, two molecular markers were further discarded as they showed high FPR. The SNP marker snp0S00939, which also showed close clustering, had an FPR of $26.1 \%$ and an FNR of $6.5 \%$. Another discarded marker was snpOS00948, which initially showed good clustering, but further analysis showed a very high FPR of $54.5 \%$ and an FNR of $3.1 \%$. In total 12 markers showed acceptable FPR and FNR and were considered good candidates to validate the effect of QTL mentioned above across multiple genetic backgrounds (Table 2). On average, the newly developed KASPbased SNP molecular markers showed excellent call rates of $99.56 \%$ and ranged from $97.87-100.00 \%$. FNR was also relatively low with an average of $2.27 \%$, ranging from $0.0-8.3 \%$. Finally, FPR ranged from 3.3-9.5\%, with an average of $7.61 \%$. In summary, nine markers (snp0S00933, snp0S00934, snpOS00935, snpOS00937, snpOS00938, snpOS00940, snpOS00941, snpOS00942, and snpOS00944) targeting $q R C A 4$ and one each for qRCA1.1 (snp0S00945), qRCA2.1 (snp0S00947), and qRCA2.2 (snpOS00948) were used to validate their respective effects across genetic backgrounds. For $q R C A 4$, the nine markers were distributed across the previously reported confidence interval of the QTL. Two of the markers, snp0S00933 and snp0S00944 were designed to be flanking the QTL, while the other seven were designed within the QTL. On average, the physical inter-marker distance was 136.7 kilobase (kb), with the highest distance ( $273.3 \mathrm{~kb}$ ) observed between snp0S00933 and snp0S00934 and the lowest (41.2 kb) between snp0S00940 and snp0S00941. 
Table 2

Molecular marker quality metrics of newly developed KASP-based SNP markers targeting qRCA QTL.

\begin{tabular}{|c|c|c|c|c|c|c|c|}
\hline SNP ID & Chr_bp & QTL & SNP & $\begin{array}{l}\text { Favourable } \\
\text { Allele }\end{array}$ & $\begin{array}{l}\text { Call } \\
\text { Rate }\end{array}$ & $\begin{array}{l}\text { FPR } \\
(\%)\end{array}$ & $\begin{array}{l}\text { FNR } \\
\text { (\%) }\end{array}$ \\
\hline snp0S00933 & Chr04_29670314 & $q R C A 4$ & $\mathrm{~T} / \mathrm{A}$ & $\mathrm{T}$ & 98.94 & 4.3 & 6.5 \\
\hline snp0S00934 & Chr04_29943687 & $q R C A 4$ & $A / C$ & $A$ & 97.87 & 8.7 & 3.2 \\
\hline snp0S00935 & Chr04_30001385 & $q R C A 4$ & $\mathrm{C} / \mathrm{T}$ & $\mathrm{C}$ & 100.00 & 8.7 & 0.0 \\
\hline snp0S00937 & Chr04_30193259 & $q R C A 4$ & $\mathrm{G} / \mathrm{C}$ & $\mathrm{G}$ & 100.00 & 8.7 & 0.0 \\
\hline snp0S00938 & Chr04_30302635 & $q R C A 4$ & $A / G$ & $A$ & 98.94 & 8.7 & 0.0 \\
\hline snpOS00940 & Chr04_30420667 & $q R C A 4$ & $A / C$ & $A$ & 100.00 & 8.7 & 0.0 \\
\hline snpOS00941 & Chr04_30461857 & $q R C A 4$ & T/A & $\mathrm{T}$ & 100.00 & 8.7 & 0.0 \\
\hline snp0S00942 & Chr04_30524467 & qRCA4 & $A / G$ & $A$ & 100.00 & 8.7 & 0.0 \\
\hline snp0S00944 & Chr04_30764226 & $q R C A 4$ & $\mathrm{G} / \mathrm{A}$ & G & 98.94 & 8.7 & 0.0 \\
\hline snp0S00945 & Chr01_39443793 & qRCA1.1 & $\mathrm{G} / \mathrm{A}$ & $A$ & 100.00 & 4.5 & 3.1 \\
\hline snpOS00947 & Chr02_27974625 & qRCA2.1 & $\mathrm{C} / \mathrm{T}$ & $\mathrm{T}$ & 100.00 & 9.5 & 6.1 \\
\hline snp0S00948 & Chr02_30450101 & $q R C A 2.2$ & $A / T$ & $A$ & 100.00 & 3.3 & 8.3 \\
\hline
\end{tabular}

Examining the genotype composition of the six varieties, the nine markers targeting qRCA4 were shown to be polymorphic between the donor IRAT109, and other genotypes - Langi, Reiziq, RL11, and Sherpa. In the case of Norin PL8, of the nine markers, six were shown to be polymorphic, while the other three (snp0S00934, snp0S00938, and snp0S00941) were monomorphic. For qRCA1.1, Reiziq and RL11, including IRAT109, showed unfavourable allele, while for $q R C A 2.1$, only IRAT109 showed unfavourable allele. Lastly, for qRCA2.2, except for IRAT109, all genotypes have unfavourable allele.

\section{QTL validation and effects across genetic backgrounds}

Three $\mathrm{F}_{2}$ populations were used to validate the effects of $q R C A \mathrm{QTL}$ in different genetic backgrounds. Of the nine qRCA4 markers developed, two (snp0S00937 and snpOS00942) were excluded from the analysis as they were calling IRAT109 as heterozygote. Downstream analysis was carried out using the remaining seven markers. Across the three populations, all the seven markers followed the expected Mendelian segregation ratio of $F_{2}\left(\chi^{2}[1: 2: 1]=0.57-4.86, P>0.05\right)$ populations (Table S1). This indicated conformance of both the molecular makers and the populations with expected ratios. Estimation of the genetic distance between the markers revealed a total of 5.70 centiMorgan (cM) distance between the two flanking markers, with an average distance of $0.97 \mathrm{cM}$ between markers. Evaluation of the effect of 
populations: IRLA and IRRL, while there was no significant association detected in the IRNO population (Table 3). Examining the individual populations, significant association was revealed in IRRL population with logarithm of odds (LOD) of 13.36. Introgression of the favourable IRAT109 allele (BB) suggested an effect of narrower RCA of about $15.12^{\circ}$. Additionally, a significant dominance effect was also detected, indicating the heterozygotes have $4.08^{\circ}$ narrower RCA than expected, based on the estimated additive effects of the IRAT109 allele. In this population, using these molecular markers, qRCA4 was also shown to explain $17.27 \%$ of the genotypic variation present in IRRL. Furthermore, the effect of $q R C A 4$ in IRLA had a LOD of 4.13 and the introgression of the IRAT109 allele showed an estimated effect of $11.87^{\circ}$ narrower RCA, compared with that of the unfavourable allele (AA). A significant dominance effect was also detected, with heterozygotes having $1.02^{\circ}$ narrower RCA and the region also explained $9.25 \%$ of the variation present in IRLA population. In terms of the third population, no significant association was detected, indicating that the other parent in this population (Norin PL8) may already possess the qRCA4 locus, which is further supported by the presence of three monomorphic markers between the parents and is also congruent with the phenotypic observations for Norin PL8's RCA carried out above.

Table 3

Validation of $q R C A 4$ effects across different genetic backgrounds using $\mathrm{F}_{2}$ populations derived from IRAT109.

\begin{tabular}{|llllll|}
\hline Population & N & LOD & AE & DE & R $^{2}$ \\
\hline IRLA & 196 & 4.13 & -5.87 & -1.02 & 9.25 \\
\hline IRRL & 331 & 13.63 & -7.56 & -4.08 & 17.27 \\
\hline IRNO & 283 & NS & NS & NS & NS \\
\hline $\begin{array}{l}\text { N- number of F } \\
\text { DE- dominance effect of the allele from IRAT109; } \text { R }^{2} \text { - percentage of genotypic variation explained by } \\
\text { QTL; NS- not significant }\end{array}$ & & & & \\
\hline
\end{tabular}

Bayesian credible confidence intervals were also estimated using the association detected in the two populations above. Using the IRRL population, the qRCA4 confidence interval was delimited between 1.13-5.70 cM, translating between 29.88-30.76 megabase (Mb) physical position of chromosome 4 . For IRLA population, $q R C A 4$ was delimited between 1.92-5.70 cM (30.04-30.76 Mb). Combining these two results, it was shown that $q R C A 4$ was fine mapped into $\sim 720 \mathrm{~kb}$ region of chromosome 4 (Fig. 1). Compared with previous results, we can now confidently indicate that this valuable genetic region is flanked between snp0S00935 and snp0S00944.

In terms of the validation of effects of $q R C A 1.1, q R C A 2.1$, and $q R C A 2.2$, SNP markers snp0S00945, snpOS00947, and snpOS00948 were used, respectively. It was noted that for IRNO, snpOS00947 did not follow expected Mendelian segregation ratio for $\left(\chi^{2}[1: 2: 1]=39.13, P<0.05\right)$ and for IRLA, both snpOS00945 and snp0S00947 also did not follow expected ratios $\left(\chi_{[1: 2: 1]}^{2}=7.08-16.35, P<0.05\right)$. On the Loading [MathJax]/jax/output/CommonHTML/fonts/TeX/fontdata.js $\left.{ }^{2}[1: 2: 1]=0.21-1.88, P>0.05\right)$. Unfortunately, 
SMA carried out using these markers indicated no significant effect in the RCA across three populations tested. With these results, no downstream analyses were carried out for these loci and SNP markers.

\section{Performance of newly developed KASP markers targeting qRCA4 locus}

Utilising the $\mathrm{F}_{2}$ SNP genotyping data, molecular marker quality metrics were analysed for the seven markers. On average, the qRCA4 targeting markers showed a call rate of $99.21 \%$, with snp0S00940 and snp0S00944 showing the highest call rates at $99.51 \%$, while snpOS00933 had $98.64 \%$ (Fig. 2). An additional 13 genotypes important to the Australian breeding program were also genotyped for $q R C A 4$ markers. This analysis showed three genotypes (55A, Moroberekan, and YRF210) possess the qRCA4 favourable alleles. Bringing these results together with the checks previously genotyped, it can be noted that the breeding program specific utility of these markers were at about $83 \%$.

\section{Identification of candidate genes and possible donors qRCA4 in $3 \mathrm{~K}$ in all subpopulations}

Through the 3KRGP, potential donors for $q R C A 4$ were identified among different rice subpopulations. Since the original donor, IRAT109, is a tropjap genotype, it was expected that there were more donors in this subpopulation. Indeed, 157 out of 372 (42.2\%) tropical japonicas possessed the favourable alleles of qRCA4. In terms of the other subpopulations, a total of 21 potential donors were identified (Table S2). A single donor, Y134, from the indica subpopulation was identified while two, IAS 22 - 8 Palmar and CX578, were identified for the tempjap subpopulation. The remaining 18 were representatives of the subtrop population, majority of which (13) originated from Lao People's Democratic Republic, two from Thailand, and a single accession from China.

Examining the annotated genes within the qRCA4 region, it was found that there were 111 genes with transcript evidence, with 22 of them having alternative transcripts or splice variants. Of the 111 genes, 83 have been identified to have known molecular and/or biological functions or have been shown to have high similarity with known proteins, while the other 28 code for hypothetical genes or non-protein coding transcript. Of those identified with known functions, four have been identified to be associated with plant response to abiotic stress stimulus (LOC_Os04g50820, LOC_Os04g50880, LOC_Os04g50990, and LOC_Os04g51330). The SNP marker, snpOS00938 sits within the sequence of LOC_Os04g51172, and is predicted to be an intron variant of the gene. Additionally, it was noted that snp0S00941 is a downstream gene variant of LOC_Os04g51440, a putative villin protein and have been shown to be important regulator of actin. It is orthologous to the Arabidopsis gene AT4G30160 with a putative villin 4 function.

\section{Discussion}

\section{qRCA4 is effective across multiple genetic backgrounds}


Due to variation in genetic backgrounds, a genomic region identified to be effective in one mapping population may not necessarily be identified in other genetic backgrounds. Validation of the effect of these QTL in multiple genetic background is necessary before more downstream work can be carried out i.e., gene cloning and integration in breeding programs. This study utilised $\mathrm{F}_{2}$ segregating populations derived from three different genetic backgrounds to validate the effect of a major genomic region, $q R C A 4$, controlling narrow root cone angle in AP systems. This region was shown to have significant effects in two (RL11 and Langi) out of three genetic backgrounds, with effects ranging from a decrease of $11.7^{\circ}-$ $15.1^{\circ}$ in RCA, explaining between $9.3-17.3 \%$ of the genetic variation present, further strengthening its significance and utility in improving rice AP adaptation. In rice, similar strategies have been carried out, such as the case of SUB1A QTL mapping and molecular breeding. SUB1 was first mapped using $\mathrm{F}_{3} \mathrm{~S}$ derived from IR40931-26/PI543581 (Xu and Mackill 1996), and then several independent studies confirmed this QTL across several genetic backgrounds (Nandi et al. 1997; Siangliw et al. 2003). These laid down the foundation towards breeding for Sub1 mega-varieties through the subsequent identification of causative gene and SNP molecular markers which enabled transfer through marker assisted selection (MAS) (Bailey-Serres et al. 2010). Following this, validation of $q R C A 4$ is therefore a critical step not only to ensure its effectiveness across several genetic backgrounds but to also warrant its utility in breeding programs through MAS. Similar to the present results, DRO2 was also detected in three genetic backgrounds, ARC5955, Pinulupot 1, and Tupa729, and was shown to be related with deeper rooting in transplanted systems (Uga et al. 2013b). In the population derived from Norin PL8, no significant association was detected although significant phenotypic variation existed in the $F_{2}$ indicating that other genomic regions may play a role in the control of RCA in this population. It can also be noted that three markers utilised in this study were monomorphic between IRAT109 and Norin PL8, in addition to Norin PL8 showing narrower RCA compared with the other checks evaluated in this study. This further supports the notion that Norin PL8 already possess the qRCA4 locus.

\section{KASP-based SNP markers tagging qRCA4 were developed}

Molecular markers are essential for the seamless introgression of target traits into recipient genotypes carried out through MAS in a typical molecular breeding program. In rice, a suite of molecular markers has been designed and developed tagging high value traits such as yield components, grain quality, and disease resistance (Steele et al. 2018; Yang et al. 2019). This study, for the first time, reports the simultaneous validation and development of KASP based SNP markers targeting qRCA4, a highly valuable genomic region for AP adaptation. With the advent of more cost-effective whole genome sequencing technologies, SNPs became the marker of choice in many breeding programs. Using the Fluidigm SNP genotyping platform, Kim et al. (2016) developed SNP markers targeting yield enhancing loci - Gn1a, SPL14, Ghd7, GS5, and GS3. Gel-based SNP markers have also been developed targeting blast and bacterial blight resistance genes, Pita and xa5, respectively (Ramkumar et al. 2015). These other SNP genotyping platforms were also available but KASP has been in the forefront of these as it is more preferred due to its cost effectivity, assay conversion rate, accuracy, and flexibility (Semagn et al. 2014), especially in the context of marker assisted backcrossing and marker assisted recurrent selection

Loading [MathJax]/jax/output/CommonHTML/fonts/TeX/fontdata.js Nork using single seed genotyping in rice,

Page 14/27 
further facilitating its adoption in breeding programs by allowing breeders to combine rapid generation advance techniques more effectively (Arbelaez et al. 2019b). To our knowledge, these are the first high throughput SNP markers targeting root growth angle traits developed, whether in traditional flooded or in AP systems. KASP markers have been developed at IRRI, but have not included root traits such as RCA. An independent work by Steele et al. (2018) also developed KASP markers but only included disease resistance and grain quality traits. Additionally, Yang et al. (2019) also developed a core KASP SNP set tailored for rice varietal assessment, genetic diversity analysis, and also included markers for agronomic traits such as yield, quality, resistance, fertility, and phenology.

Using the newly developed KASP markers, this present study also further delimited the confidence interval of the qRCA4 QTL into $\sim 720 \mathrm{~kb}$ region, from a previous $\sim 910 \mathrm{~kb}$ region. This new confidence interval is flanked by markers snp0S00935 and snpOS00944. Additionally, SNP markers snpOS00938 and snp0S00941, may also be the most useful markers as they may represent the variation that is indicative of the presence of $q R C A 4$. In terms of $q R C A 1.1, q R C A 2.1$, and $q R C A 2.2$, no markers were developed associated with these as the markers used did not show significant association with RCA in the tested populations. This may be due to several reasons. First, the markers tested were very limited, only one each for the individual QTL and may warrant the design of additional markers to saturate each region. Second is that these QTL might be background specific which further highlights the importance of this QTL validation exercise. Lastly, in terms of $q R C A 2.1$ and $q R C A 2.2$, the markers targeting them may have not shown effect since these QTL were only shown to be significant in IWS conditions (Vinarao et al. 2021). Further testing of the designed markers in IWS conditions should be carried out to definitively check the association of these markers with RCA.

Examining the LD map for $q R C A 4$, the four markers identified above represent the three linkage groups detected and are therefore enough to ascertain the introgression of the qRCA4 locus. Molecular marker quality metrics (as described by Platten et al. (2019)) of these four markers also showed high call rates, low FNR, and relatively low FPR. By genotyping lines relevant to the Australian breeding program, it has also shown the predicted high utility of these markers. The KASP assay, compared with other fixed SNP arrays, are flexible and individual markers can be run together with other markers. By developing $q R C A 4$ markers in KASP system, this also ensures its seamless integration in breeding programs and can be easily pyramided with other high-value AP adaptation genes/QTL such as those for cold tolerance.

\section{KRGP and database search facilitated the identification of potential donors and candidate genes}

In any breeding program, the availability of donor and favourable alleles for traits of interest is very important. In cases where these alleles/traits are not available, exotic germplasm such as wild progenitors may be used but this would take an arduous effort to transfer the traits into cultivated germplasm. It is therefore highly important to identify readily available donor genotypes for use in specific breeding programs. Using the publicly available 3KRGP SNP genotyping data (Mansueto et al. 2017). allele minina was carried out to identifv possible donors within specific subpopulations. Donors in Loading [MathJax]/jax/output/CommonHTML/fonts/TeX/fontdata.js

Page $15 / 27$ 
various subpopulations were identified including indica, tempjap, tropjap, and subtrop. These donors are available at IRRI and may be requested if deemed useful for the particular program. This study also highlights the utility of publicly available databases to augment in discovery process and help breeders make more informed decisions, as in this case, donor parent selection.

Using the newly determined confidence interval, candidate genes along the $q R C A 4$ region were also identified through RAPDB (Sakai et al. 2013). Genes related with plant response to abiotic stress stimulus were identified, including LOC_Os04g51330. LOC_Os04g51330 was similar to a maltose excess protein, a probable maltose transporter vital for the conversion of starch to sucrose in leaves at night (Niittyla et al. 2004) and more importantly, an orthologue of the Arabidopsis root cap protein 1. On the other hand, LOC_Os04g51440, was identified which is an orthologue of to the Arabidopsis gene AT4G30160 (putative VILLIN 4). In Arabidopsis, VILLIN 4 proteins have been to be involved in root hair growth through the regulation of actin organisation (Zhang et al. 2011), and in rice, VILLIN 2 was shown to be important in regulating plant architecture through the modulation of microfilament dynamics and polar auxin transport (Wu et al. 2015). These genes represent excellent targets for downstream cloning experiments to characterise the molecular function of the gene associated with qRCA4 and narrow RCA in AP systems. Emerging technologies for gene cloning such as CRISPR (Clustered Regularly Interspaced Short Palindromic Repeats) with CRISPR-associated protein Cas9 (CRISPR-Cas9) holds promise and may be utilised to precisely engineer and target variations present in the $q R C A 4$ region and candidate genes of interest (Zafar et al. 2020). Non-targeted metabolic profiling of genotypes with contrasting RCA phenotypes and $q R C A$ composition across several environmental conditions may also reveal biochemical pathways and specific metabolites which may aid in understanding mechanisms related with rice plant's ability to cope with and tolerate stress involving transient water deficit. Finally, transcriptomic profiling and network analysis of contrasting genotypes may show differentially expressed genes in modules and along with metabolic profiling, may provide a systems level understanding of rice response to transient water deficit, and ultimately, AP adaptation.

\section{Conclusions}

This study validated the effect of $q R C A 4$ in multiple genetic backgrounds further reinforcing its value in improvement of rice genotypes for AP adaptation. Simultaneously, high-throughput KASP SNP molecular markers were also developed to tag this genomic region which will facilitate its seamless introgression into target recipient genotypes especially in breeding programs where AP adaptation traits are important. This study reports for the first time the development of such markers associated with root traits, whether in traditional transplanted flooded or in AP systems. Molecular marker quality metrics also show marker robustness and possible high utility in breeding programs. Using 3KRGP, potential donors across subpopulations were identified representing readily available donors for use of prospective breeding programs. Candidate genes along the qRCA4 region were also identified and were shown to either be associated with plant response to abiotic stress stimulus or related with root development. Physiological analysis of the relationship of RCA and this locus with grain yield and key traits such as those related to

Loading [MathJax]/jax/output/CommonHTML/fonts/TeX/fontdata.js ent environmental conditions will shed some 
light on mechanisms related to its action and further increase the value of this genomic region and associated molecular markers, specifically in AP systems. This study provides the tools to breeders for the development of genotypes with narrow rooting angle and greater depth, through MAS, ultimately for more sustainable rice production in environments where rice is exposed to transient water deficit.

\section{Abbreviations}

3KRGP

3K Rice Genome Project

AP

aerobic rice production

DAS

days after sowing

FNR

false negative rate

FPR

false positive rate

IRLA

IRAT109/Langi

IRNO

IRAT109/Norin PL8

IRRI

International Rice Research Institute

IRRL

IRAT109/RL 11

IWS

intermittent water stress

KASP

kompetive allele-specific PCR

LD

linkage disequilibrium

LOD

logarithm of odds

MAS

marker assisted selection

QTL

quantitative trait loci

RAP-DB

Rice Annotation Project Database

\section{RrA}


root cone angle

RDR

ratio of deep roots

RIL

recombinant inbred lines

RP

recipient parent

RSA

root system architecture

SMA

single marker analysis

SNP

single nucleotide polymorphism

SSR

simple sequence repeats

UQ

University of Queensland

\section{Declarations}

\section{Funding}

This work was funded by AgriFutures under project number: PRJ-011067.

\section{Competing interests}

The authors declare no conflicts of interest in this study.

\section{Ethics approval and consent to participate}

Not applicable

Consent for publication

Not applicable

\section{Availability of Data and Materials}

The datasets used and/or analysed during the current study are available from the corresponding author 


\section{Code availability}

Not applicable

\section{Authors' contributions}

All authors contributed to the study design. C.P. developed the segregating populations utilised in this paper. R.V. carried out glasshouse experiments, R.V. and C.P. performed data analysis and linkage mapping. The first draft of the manuscript was written by R.V. and all authors commented on subsequent versions of the manuscript. All authors read and approved the final manuscript.

\section{Acknowledgments}

The authors would like to thank the UQ's Central Glasshouse Services, Dr. Lisa Xian, and Dr. Venea Dara Daygon for their technical support. The authors would also like to thank the New South Wales Department of Primary Industries for providing the fixed genotypes utilised in this study. R.V. was supported by the Australian Government Research Training Program Scholarship and the Charles Morphett Pegler Scholarship.

\section{References}

Arbelaez JD et al. (2019a) 1k-RiCA (1K-Rice Custom Amplicon) a novel genotyping amplicon-based SNP assay for genetics and breeding applications in rice. Rice 12:55. doi:10.1186/s12284-019-0311-0

Arbelaez JD et al. (2019b) Methodology: ssb-MASS: a single seed-based sampling strategy for markerassisted selection in rice. Plant Methods 15. doi:10.1186/s13007-019-0464-2

Bailey-Serres J, Fukao T, Ronald P, Ismail A, Heuer S, Mackill D (2010) Submergence tolerant rice: SUB1's journey from landrace to modern cultivar. Rice 3:138-147. doi:10.1007/s12284-010-9048-5

Bradbury PJ, Zhang Z, Kroon DE, Casstevens TM, Ramdoss Y, Buckler ES (2007) TASSEL: software for association mapping of complex traits in diverse samples. Bioinformatics 23:2633-2635

Broman KW, Wu H, Sen S, Churchill GA (2003) R/qtl: QTL mapping in experimental crosses.

Bioinformatics 19:889-890. doi:10.1093/bioinformatics/btg112

Cobb JN, Biswas PS, Platten JD (2019) Back to the future: revisiting MAS as a tool for modern plant breeding. Theor Appl Genet 132:647-667. doi:10.1007/s00122-018-3266-4

Collard BCY, Mackill DJ (2008) Marker-assisted selection: an approach for precision plant breeding in the twenty-first century. Philos Trans R Soc B-Biol Sci 363:557-572. doi:10.1098/rstb.2007.2170 
Elliott $J$ et al. (2014) Constraints and potentials of future irrigation water availability on agricultural production under climate change. Proc Natl Acad Sci U S A 111:3239-3244.

doi:10.1073/pnas.1222474110

Kato Y, Abe J, Kamoshita A, Yamagishi J (2006) Genotypic variation in root growth angle in rice (Oryza sativa L.) and its association with deep root development in upland fields with different water regimes. Plant Soil 287:117-129. doi:10.1007/s11104-006-9008-4

Kato Y, Katsura K (2014) Rice adaptation to aerobic soils: physiological considerations and implications for agronomy. Plant Prod Sci 17:1-12. doi:10.1626/pps.17.1

Kato Y, Okami M, Katsura K (2009) Yield potential and water use efficiency of aerobic rice (Oryza sativa L.) in Japan. Field Crop Res 113:328-334. doi:10.1016/j.fcr.2009.06.010

Khush GS (2005) What it will take to feed 5.0 billion rice consumers in 2030. Plant molecular biology 59:1-6. doi:10.1007/s11103-005-2159-5

Kim SR et al. (2016) Development and validation of allele-specific SNP/indel markers for eight yieldenhancing genes using whole-genome sequencing strategy to increase yield potential of rice, Oryza sativa L. Rice 9. doi:10.1186/s12284-016-0084-7

Kitomi Y, Kanno N, Kawai S, Mizubayashi T, Fukuoka S, Uga Y (2015) QTLs underlying natural variation of root growth angle among rice cultivars with the same functional allele of DEEPER ROOTING 1. Rice 8:12. doi:10.1186/s12284-015-0049-2

Kumar S, Stecher G, Li M, Knyaz C, Tamura K (2018) MEGA X: Molecular Evolutionary Genetics Analysis across computing platforms. Molecular Biology and Evolution 35:1547-1549.

doi:10.1093/molbev/msy096

Lou QJ et al. (2015) Quantitative trait locus mapping of deep rooting by linkage and association analysis in rice. J Exp Bot 66:4749-4757. doi:10.1093/jxb/erv246

Mackill DJ, McNally KL (2004) A model crop species: Molecular markers in rice. In: Molecular marker systems in plant breeding and crop improvement. Springer, pp 39-54

Mansueto L et al. (2017) Rice SNP-seek database update: new SNPs, indels, and queries. Nucleic Acids Res 45:D1075-D1081. doi:10.1093/nar/gkw1135

McCouch SR et al. (2002) Development and mapping of 2240 new SSR markers for rice (Oryza sativa L.). DNA Res 9:199-207. doi:10.1093/dnares/9.6.199

Mitchell JH, Proud C, Nguyen TM, Fukai S (2019) Traits of importance for aerobic rice Paper presented at the Proceedings of the 19th Australian Society of Agronomy Conference, Wagga Wagga, NSW, Australia, 
Nandi S, Subudhi PK, Senadhira D, Manigbas NL, Sen-Mandi S, Huang N (1997) Mapping QTLs for submergence tolerance in rice by AFLP analysis and selective genotyping. Molecular and General Genetics MGG 255:1-8. doi:10.1007/s004380050468

Niittyla T, Messerli G, Trevisan M, Chen J, Smith AM, Zeeman SC (2004) A previously unknown maltose transporter essential for starch degradation in leaves. Science 303:87-89. doi:10.1126/science.1091811

Platten JD, Cobb JN, Zantua RE (2019) Criteria for evaluating molecular markers: Comprehensive quality metrics to improve marker-assisted selection. PLoS One 14:e0210529.

doi:10.1371/journal.pone.0210529

R Core Team (2019) R: A language and environment for statistical computing. R Foundation for Statistical Computing.

Ramkumar G, Prahalada GD, Hechanova SL, Vinarao R, Jena KK (2015) Development and validation of SNP-based functional codominant markers for two major disease resistance genes in rice (O. sativa L.). Mol Breed 35:129. doi:10.1007/s11032-015-0323-4

Sakai H et al. (2013) Rice Annotation Project Database (RAP-DB): An integrative and interactive database for rice genomics. Plant Cell Physiol 54:E6. doi:10.1093/pcp/pcs183

Semagn K, Babu R, Hearne S, Olsen M (2014) Single nucleotide polymorphism genotyping using Kompetitive Allele Specific PCR (KASP): overview of the technology and its application in crop improvement. Mol Breed 33:1-14. doi:10.1007/s11032-013-9917-x

Siangliw M, Toojinda T, Tragoonrung S, Vanavichit A (2003) Thai jasmine rice carrying QTLch9 (SubQTL) is submergence tolerant. Ann Bot 91:255-261. doi:10.1093/aob/mcf123

Smith AB, Liw P, Cullis BR (2006) The design and analysis of multi-phase plant breeding experiments. Journal of Agricultural Science 144:393-409. doi:10.1017/s0021859606006319

Steele KA et al. (2018) Accelerating public sector rice breeding with high-density KASP markers derived from whole genome sequencing of indica rice. Mol Breed 38. doi:10.1007/s11032-018-0777-2

Thomson MJ et al. (2017) Large-scale deployment of a rice 6 K SNP array for genetics and breeding applications. Rice 10:40. doi:10.1186/s12284-017-0181-2

Uga Y, Kitomi Y, Yamamoto E, Kanno N, Kawai S, Mizubayashi T, Fukuoka S (2015) A QTL for root growth angle on rice chromosome 7 is involved in the genetic pathway of DEEPER ROOTING 1. Rice 8. doi:10.1186/s12284-015-0044-7

Uga $Y$ et al. (2013a) Control of root system architecture by DEEPER ROOTING 1 increases rice yield under drought conditions. Nature Genet 45:1097. doi:10.1038/ng.2725 
Uga Y, Yamamoto E, Kanno N, Kawai S, Mizubayashi T, Fukuoka S (2013b) A major QTL controlling deep rooting on rice chromosome 4. Sci Rep 3. doi:10.1038/srep03040

Vinarao R, Proud C, Zhang X, Snell P, Fukai S, Mitchell J (2021) Stable and novel quantitative trait loci (QTL) confer narrow root cone angle in an aerobic rice (Oryza sativa L.) production system. Rice 14:28. doi:10.1186/s12284-021-00471-2

Wu SY et al. (2015) VLN2 regulates plant architecture by affecting microfilament dynamics and polar auxin transport in rice. Plant Cell 27:2829-2845. doi:10.1105/tpc.15.00581

Xu K, Mackill DJ (1996) A major locus for submergence tolerance mapped on rice chromosome 9. Mol Breed 2:219-224. doi:10.1007/BF00564199

Yang G et al. (2019) Development of a core SNP arrays based on the KASP method for molecular breeding of rice. Rice 12:21. doi:10.1186/s12284-019-0272-3

Zafar K et al. (2020) Genome editing technologies for rice improvement: progress, prospects, pand safety concerns. Frontiers in Genome Editing 2:5. doi:10.3389/fgeed.2020.00005

Zhang Y, Xiao YY, Du F, Cao LJ, Dong HJ, Ren H (2011) Arabidopsis VILLIN4 is involved in root hair growth through regulating actin organization in a Ca2+-dependent manner. New Phytol 190:667-682. doi:10.1111/j.1469-8137.2010.03632.x

\section{Supplementary Tables}

Table S1 Chi-square analysis of the observed and expected Mendelian segregation ratio for unfavourable $(A A)$, favourable (BB), and heterozygote (AB) alleles in the $F_{2}$ populations (POP). 


\begin{tabular}{|lllllll|}
\hline SNP ID & POP & AA & AB & BB & Chi-Square & P Value \\
\hline snpOS00933 & IRLA & 50 & 101 & 42 & 1.08 & 0.582 \\
\hline snpOS00934 & IRLA & 49 & 103 & 43 & 0.99 & 0.610 \\
\hline snpOS00935 & IRLA & 49 & 104 & 41 & 1.67 & 0.434 \\
\hline snpOS00938 & IRLA & 44 & 109 & 43 & 2.48 & 0.289 \\
\hline snpOS00940 & IRLA & 44 & 107 & 45 & 1.66 & 0.435 \\
\hline snpOS00941 & IRLA & 44 & 107 & 45 & 1.66 & 0.435 \\
\hline snpOS00944 & IRLA & 44 & 106 & 46 & 1.35 & 0.510 \\
\hline snpOS00945 & IRLA & 47 & 111 & 33 & 7.08 & $0.029 *$ \\
\hline snpOS00947 & IRLA & 46 & 118 & 25 & 16.35 & $0.000 *$ \\
\hline snpOS00948 & IRLA & 54 & 91 & 48 & 1.00 & 0.607 \\
\hline snpOS00933 & IRNO & 65 & 134 & 82 & 2.66 & 0.265 \\
\hline snpOS00935 & IRNO & 66 & 137 & 80 & 1.67 & 0.434 \\
\hline snpOS00940 & IRNO & 65 & 138 & 79 & 1.52 & 0.468 \\
\hline snpOS00944 & IRNO & 66 & 141 & 75 & 0.57 & 0.750 \\
\hline snpOS00945 & IRNO & 70 & 151 & 58 & 2.93 & 0.231 \\
\hline snpOS00947 & IRNO & 70 & 179 & 26 & 39.13 & $0.000 *$ \\
\hline snpOS00948 & IRNO & 61 & 146 & 76 & 1.88 & 0.391 \\
\hline snpOS00933 & IRRL & 87 & 171 & 67 & 3.35 & 0.187 \\
\hline snpOS00934 & IRRL & 89 & 166 & 69 & 2.67 & 0.264 \\
\hline snpOS00935 & IRRL & 89 & 168 & 71 & 2.17 & 0.338 \\
\hline snpOS00938 & IRRL & 92 & 168 & 65 & 4.86 & 0.088 \\
\hline snpOS00940 & IRRL & 91 & 170 & 67 & 3.95 & 0.139 \\
\hline snpOS00941 & IRRL & 91 & 168 & 67 & 3.84 & 0.147 \\
\hline snpOS00944 & IRRL & 89 & 170 & 69 & 2.88 & 0.237 \\
\hline snpOS00947 & IRRL & 74 & 169 & 65 & 3.45 & 0.178 \\
\hline snpOS00948 & IRRL & 77 & 155 & 82 & 0.21 & 0.900 \\
\hline
\end{tabular}

*: $P<0.05$ 


\begin{tabular}{|c|c|c|c|c|c|}
\hline Genotype/Variety & $\begin{array}{l}\text { 3KRGP } \\
\text { IRIS ID }\end{array}$ & $\begin{array}{l}\text { Country } \\
\text { of Origin }\end{array}$ & Chr04:30302635 & Chr04:30461857 & Subpopulation \\
\hline Y134 & CX15 & - & A & $\mathrm{T}$ & Indica \\
\hline $\begin{array}{l}\text { IAS 22-8 } \\
\text { PALMAR::IRGC } \\
26058-1\end{array}$ & $\begin{array}{l}\text { IRIS } \\
313- \\
10967\end{array}$ & Brazil & A & $\mathrm{T}$ & $\begin{array}{l}\text { Temperate } \\
\text { japonica }\end{array}$ \\
\hline CX578 & CX578 & - & A & $\mathrm{T}$ & $\begin{array}{l}\text { Temperate } \\
\text { japonica }\end{array}$ \\
\hline $\begin{array}{l}\text { LEUANG } \\
\text { GLIANG::IRGC } \\
71271-1\end{array}$ & $\begin{array}{l}\text { IRIS } \\
313- \\
9123\end{array}$ & Thailand & A & $\mathrm{T}$ & $\begin{array}{l}\text { Subtropical } \\
\text { japonica }\end{array}$ \\
\hline $\begin{array}{l}\text { NAM ROO::IRGC } \\
66996-1\end{array}$ & $\begin{array}{l}\text { IRIS } \\
313- \\
10176\end{array}$ & Thailand & A & $\mathrm{T}$ & $\begin{array}{l}\text { Subtropical } \\
\text { japonica }\end{array}$ \\
\hline CHA LOY OE::C1 & $\begin{array}{l}\text { IRIS } \\
313- \\
7856\end{array}$ & - & A & $\mathrm{T}$ & $\begin{array}{l}\text { Subtropical } \\
\text { japonica }\end{array}$ \\
\hline $\begin{array}{l}\text { KAO SONG } \\
\text { HAI::IRGC 61827-1 }\end{array}$ & $\begin{array}{l}\text { IRIS } \\
313- \\
11623\end{array}$ & China & A & $\mathrm{T}$ & $\begin{array}{l}\text { Subtropical } \\
\text { japonica }\end{array}$ \\
\hline $\begin{array}{l}\text { SI NAM } \\
\text { PUENG::IRGC } \\
\text { 75165-1 }\end{array}$ & $\begin{array}{l}\text { IRIS } \\
313- \\
11926\end{array}$ & Thailand & A & $\mathrm{T}$ & $\begin{array}{l}\text { Subtropical } \\
\text { japonica }\end{array}$ \\
\hline $\begin{array}{l}\text { XIENG } \\
\text { KANG::IRGC } \\
82533-1\end{array}$ & $\begin{array}{l}\text { IRIS } \\
313- \\
12063\end{array}$ & Lao PDR & A & $\mathrm{T}$ & $\begin{array}{l}\text { Subtropical } \\
\text { japonica }\end{array}$ \\
\hline $\begin{array}{l}\text { THATENG::IRGC } \\
86377-1\end{array}$ & $\begin{array}{l}\text { IRIS } \\
313- \\
12134\end{array}$ & Lao PDR & A & $\mathrm{T}$ & $\begin{array}{l}\text { Subtropical } \\
\text { japonica }\end{array}$ \\
\hline $\begin{array}{l}\text { SET::IRGC 92200- } \\
1\end{array}$ & $\begin{array}{l}\text { IRIS } \\
313- \\
12227\end{array}$ & Lao PDR & A & $\mathrm{T}$ & $\begin{array}{l}\text { Subtropical } \\
\text { japonica }\end{array}$ \\
\hline $\begin{array}{l}\text { A MOUK::IRGC } \\
94474-1\end{array}$ & $\begin{array}{l}\text { IRIS } \\
313- \\
12252\end{array}$ & Lao PDR & A & $\mathrm{T}$ & $\begin{array}{l}\text { Subtropical } \\
\text { japonica }\end{array}$ \\
\hline $\begin{array}{l}\text { BAN BONG::IRGC } \\
94565-1\end{array}$ & $\begin{array}{l}\text { IRIS } \\
313- \\
12254\end{array}$ & Lao PDR & A & $\mathrm{T}$ & $\begin{array}{l}\text { Subtropical } \\
\text { japonica }\end{array}$ \\
\hline $\begin{array}{l}\text { NYAE::IRGC } \\
95302-1\end{array}$ & $\begin{array}{l}\text { IRIS } \\
313- \\
12262\end{array}$ & Lao PDR & A & $\mathrm{T}$ & $\begin{array}{l}\text { Subtropical } \\
\text { japonica }\end{array}$ \\
\hline TA SENG::IRGC & IRIS & Lao PDR & A & \multirow[t]{2}{*}{$\mathrm{T}$} & \multirow{2}{*}{$\begin{array}{l}\text { Subtropical } \\
\text { japonica }\end{array}$} \\
\hline Loading [MathJax]/jax/c & $\mathrm{ut} / \mathrm{Commc}$ & $\mathrm{ML} /$ fonts/T & & & \\
\hline
\end{tabular}




\begin{tabular}{|c|c|c|c|c|c|}
\hline $\begin{array}{l}\text { BAN KEO::IRGC } \\
99953-1\end{array}$ & $\begin{array}{l}\text { IRIS } \\
313- \\
12323\end{array}$ & Lao PDR & A & $\mathrm{T}$ & $\begin{array}{l}\text { Subtropical } \\
\text { japonica }\end{array}$ \\
\hline $\begin{array}{l}\text { DENG NYAY::IRGC } \\
106907-1\end{array}$ & $\begin{array}{l}\text { IRIS } \\
313- \\
12332\end{array}$ & Lao PDR & A & $\mathrm{T}$ & $\begin{array}{l}\text { Subtropical } \\
\text { japonica }\end{array}$ \\
\hline $\begin{array}{l}\text { HAI NA::IRGC } \\
\text { 107117-1 }\end{array}$ & $\begin{array}{l}\text { IRIS } \\
313- \\
12342\end{array}$ & Lao PDR & A & $\mathrm{T}$ & $\begin{array}{l}\text { Subtropical } \\
\text { japonica }\end{array}$ \\
\hline $\begin{array}{l}\text { LAB::IRGC } \\
107468-1\end{array}$ & $\begin{array}{l}\text { IRIS } \\
313- \\
12346\end{array}$ & Lao PDR & A & $\mathrm{T}$ & $\begin{array}{l}\text { Subtropical } \\
\text { japonica }\end{array}$ \\
\hline $\begin{array}{l}\text { KHAI DO::IRGC } \\
12903-2\end{array}$ & $\begin{array}{l}\text { IRIS } \\
313- \\
10679\end{array}$ & Lao PDR & A & $\mathrm{T}$ & $\begin{array}{l}\text { Subtropical } \\
\text { japonica }\end{array}$ \\
\hline $\begin{array}{l}\text { KHAO TAM::IRGC } \\
30085-2\end{array}$ & $\begin{array}{l}\text { IRIS } \\
313- \\
11093\end{array}$ & Lao PDR & A & $\mathrm{T}$ & $\begin{array}{l}\text { Subtropical } \\
\text { japonica }\end{array}$ \\
\hline
\end{tabular}

Table S2. Potential donors of $q R C A 4$ locus identified using SNP genotype data publicly available through the 3K Rice Genotyping Project (3KRGP).

\section{Figures}




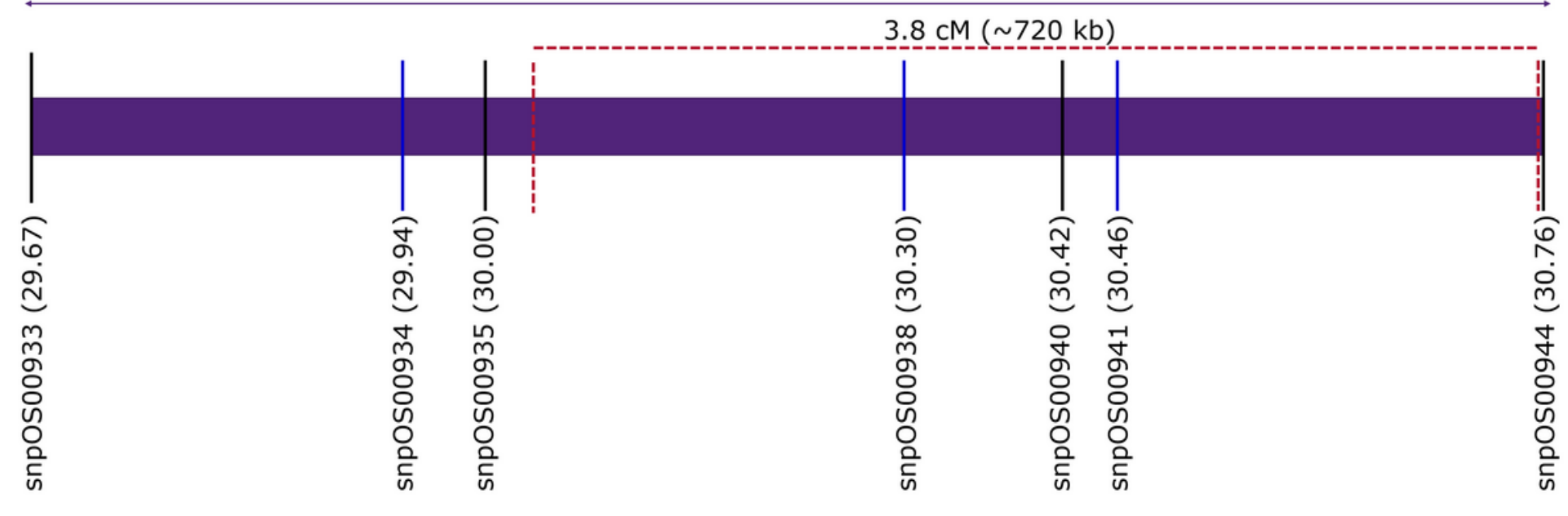

\section{Figure 1}

Molecular map of qRCA4 using newly designed molecular markers and validation results from two populations. Black lines indicate polymorphic markers between IRAT109 and other check genotypes used, while blue lines indicate monomorphic markers between IRAT109 and Norin PL8. Red dashed lines indicate the new confidence interval computed. 


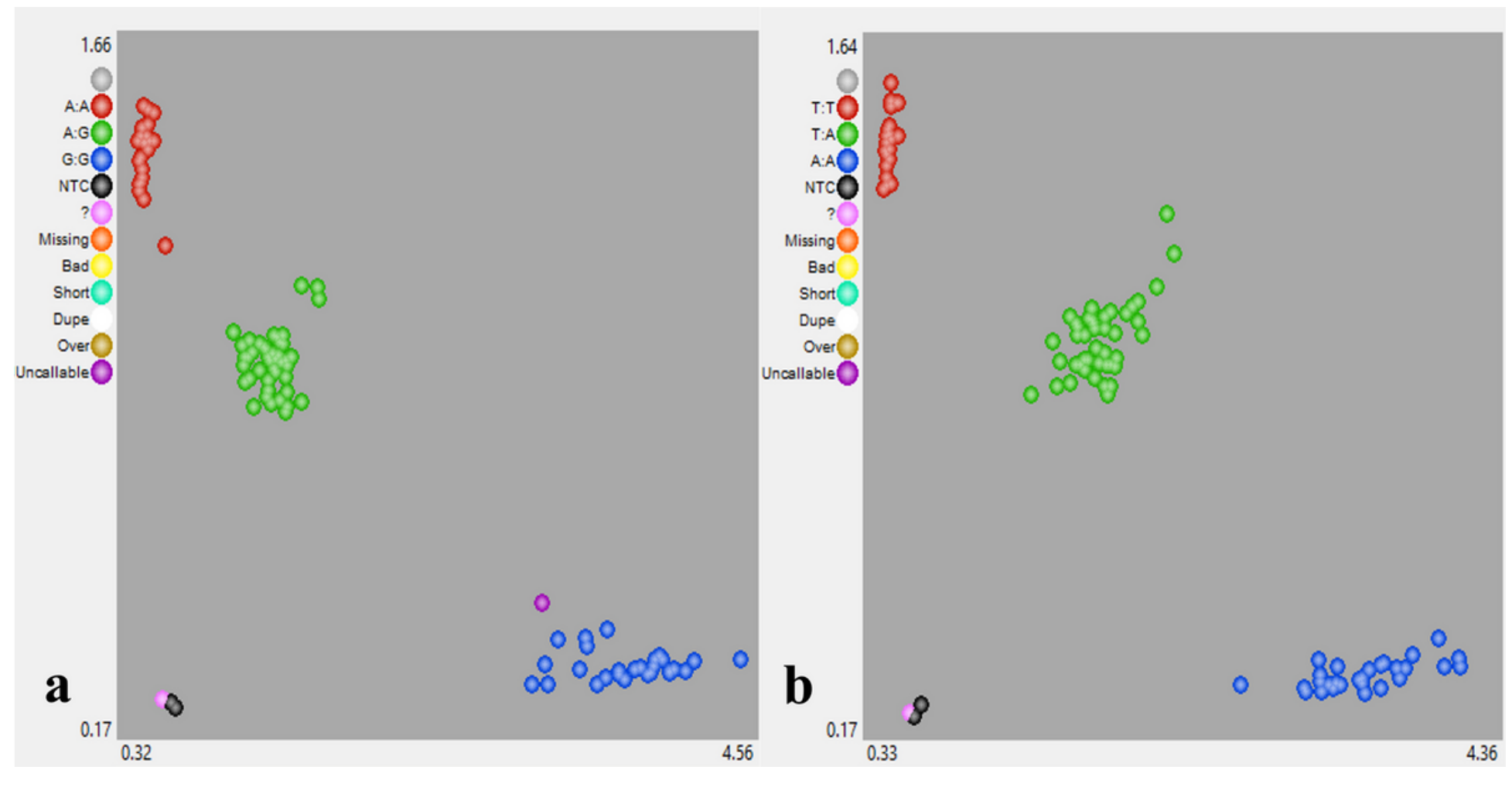

Figure 2

Cluster genotype of KASP molecular markers (A) snp0S00938 and (B) snp0S00941 using F2 segregating population.

\section{Supplementary Files}

This is a list of supplementary files associated with this preprint. Click to download.

- Fig.S1.png

- Fig.S2.png 